\title{
Efficacy of Intratracheal Budesonide-Surfactant Combined Therapy in Surfactant-Insufficient Rat Lungs with Lipopolysaccharide Insult
}

\author{
Pei-Chen Tsao ${ }^{1}$, Chih-Hsueh Lin $^{2}$, Yu-Sheng Lee ${ }^{1}$, Wei-Yu Chen ${ }^{3}$, Mei-Jy Jeng ${ }^{4}$, and Yu \\ $\mathrm{Ru} \mathrm{Kou}{ }^{4}$ \\ ${ }^{1}$ Taipei Veterans General Hospital \\ ${ }^{2}$ HungKuang University \\ ${ }^{3}$ National Yang-Ming University School of Medicine \\ ${ }^{4}$ National Yang-Ming University
}

March 10, 2021

\begin{abstract}
Objectives: Intratracheal steroid therapy for lipopolysaccharide (LPS)-induced acute lung injury (ALI) remains challenging particularly in surfactant-deficiency lungs, a common problem of preterm infants. Surfactant has been used as a vehicle for intratracheal steroid in the treatment of other types of ALI. This study investigated the efficacy of intratracheal budesonide (BUD) delivered by two concentrations of surfactant in the treatment of LPS-induced ALI in surfactant-insufficiency rat lungs. Methods: Male adult rats were anesthetized and ventilated. Our ALI model was established by repeated saline lavage to produce surfactant insufficiency, followed by intratracheal LPS instillation. Five study groups ( $\mathrm{n}=5$ for each) with different intratracheal treatments following ALI were used: Control (no treatment), BUD (IT-NS-BUD; BUD in saline); IT-DS-BUD (BUD in diluted surfactant); IT-FS-BUD (BUD in full-strength surfactant); IT-FS (full-strength surfactant). Cardiopulmonary variables were monitored $4 \mathrm{~h}$ post injury. Histological and immunohistochemical assessments of the lungs were performed. Results: The IT-FS-BUD and IT-FS groups presented better gas exchange, less metabolic acidosis, less oxygen index, and more stable hemodynamic changes than the IT-DS-BUD, IT-NS-BUD, and Control groups. The total lung injury scores assessed by histological examination were ordered as follows: IT-FS-BUD $<$ IT-DS-BUD or IT-FS $<$ IT-NS-BUD $<$ Control. The immunostaining intensities of lung myeloperoxidase showed the following order: IT-NS-BUD, IT-DS-BUD, or IT-FS-BUD < Control or IT-FS. Only the IT-FS-BUD group displayed a smaller immunostaining intensity of lung TNF- $\alpha$ than the control group. Conclusion: Among our therapeutic strategies, intratracheal BUD delivered by full-strength surfactant confers an optimal protection against LPS-induced ALI in surfactant-insufficiency rat lungs.
\end{abstract}

Efficacy of Intratracheal Budesonide-Surfactant Combined Therapy in Surfactant-Insufficient Rat Lungs with Lipopolysaccharide Insult

Pei-Chen Tsao, MD, MS,${ }^{1,2,3,6}$ Chih-Hsueh Lin, PhD,${ }^{2,4}$ Yu-Sheng Lee, MD, PhD, ${ }^{1,3,6}$ Wei-Yu Chen, MD, MS, ${ }^{1,3,5,6}$ Mei-Jy Jeng, MD, PhD, ${ }^{1,3,5,6^{*}}$ Yu Ru Kou, PhD, ${ }^{2,5,6^{*}}$

${ }^{1}$ Department of Pediatrics, Taipei Veterans General Hospital, Taipei, Taiwan.

${ }^{2}$ Institute of Physiology, School of Medicine, National Yang Ming Chiao Tung University, Taipei, Taiwan.

${ }^{3}$ Department of Pediatrics, School of Medicine, National Yang Ming Chiao Tung University, Taipei, Taiwan.

${ }^{4}$ Department of Nutrition, Master Program of BioMedical Nutrition, HungKuang University, Taichung, Taiwan. 
${ }^{5}$ Institute of Emergency and Critical Care Medicine, School of Medicine, National Yang Ming Chiao Tung University, Taipei, Taiwan.

${ }^{6}$ School of Medicine, National Yang-Ming University, Taipei, Taiwan

Running head: Budesonide-surfactant combined therapy in acute lung injury

Yu Ru Kou and Mei-Jy Jeng contributed equally.

\section{*Correspondence}

Yu Ru Kou, PhD; Institute of Physiology, School of Medicine, National Yang Ming Chiao Tung, Taipei, Taiwan. No.155, Section 2, Linong Street, Taipei, Taiwan 11221; Tel.: +886-2-2826-7080; Fax: +886-22826-4049

Email: yrkou@ym.edu.tw

Mei-Jy Jeng, MD, PhD, Department of Pediatrics, Children's Medical Center, Taipei

Veterans General Hospital, No. 201, Section 2, Shih-Pai Road, Taipei, Taiwan 11217.

Email: mjjeng@vghtpe.gov.tw

\section{Funding information}

Ministry of Science and Technology, Grant numbers: MOST 105-2314-B-010-044 and MOST 107-2320-B010-027-MY3; Taipei Veterans General Hospital, Grant number: V110C-070 and V110C-170.

\section{Abstract (250 words)}

Objectives: Intratracheal steroid therapy for lipopolysaccharide (LPS)-induced acute lung injury (ALI) remains challenging particularly in surfactant-deficiency lungs, a common problem of preterm infants. Surfactant has been used as a vehicle for intratracheal steroid in the treatment of other types of ALI. This study investigated the efficacy of intratracheal budesonide (BUD) delivered by two concentrations of surfactant in the treatment of LPS-induced ALI in surfactant-insufficiency rat lungs.

Methods: Male adult rats were anesthetized and ventilated. Our ALI model was established by repeated saline lavage to produce surfactant insufficiency, followed by intratracheal LPS instillation. Five study groups ( $\mathrm{n}=5$ for each) with different intratracheal treatments following ALI were used: Control (no treatment), BUD (IT-NS-BUD; BUD in saline); IT-DS-BUD (BUD in diluted surfactant); IT-FS-BUD (BUD in full-strength surfactant); IT-FS (full-strength surfactant). Cardiopulmonary variables were monitored $4 \mathrm{~h}$ post injury. Histological and immunohistochemical assessments of the lungs were performed.

Results: The IT-FS-BUD and IT-FS groups presented better gas exchange, less metabolic acidosis, less oxygen index, and more stable hemodynamic changes than the IT-DS-BUD, IT-NS-BUD, and Control groups. The total lung injury scores assessed by histological examination were ordered as follows: IT-FS-BUD < IT-DS-BUD or IT-FS $<$ IT-NS-BUD $<$ Control. The immunostaining intensities of lung myeloperoxidase showed the following order: IT-NS-BUD, IT-DS-BUD, or IT-FS-BUD $<$ Control or IT-FS. Only the IT-FSBUD group displayed a smaller immunostaining intensity of lung TNF- $\alpha$ than the control group.

Conclusion: Among our therapeutic strategies, intratracheal BUD delivered by full-strength surfactant confers an optimal protection against LPS-induced ALI in surfactant-insufficiency rat lungs.

Keywords: budesonide, surfactant, intratracheal instillation, surfactant-deficiency, lipopolysaccharide, acute lung injury

\section{1 | INTRODUCTION}

Pediatric pneumonia is a major cause of mortality and morbidity in children under 5 years old, especially in developing countries. ${ }^{1,2}$ Among the cases of pediatric pneumonia, $21.7 \%$ occur during the neonatal stage. ${ }^{3}$ Preterm infants who have pneumonia may develop acute lung injury (ALI) and its severe form-acute 
respiratory distress syndrome (ARDS). ${ }^{4,5}$ One major presentation of this form of ALI is lung inflammation mainly induced by lipopolysaccharide (LPS), a major pathogenic component of Gram-negative bacilli. ${ }^{6}$ In particular, the inflammatory effect of the LPS insult accompanies the injurious impact of surfactant deficiency in the lungs, a situation that generally occurs in preterm infants. ${ }^{7,8}$ Thus, surfactant deficiency and the lack of ability to cope with inflammation are the major disadvantages of these infants against LPSinduced ALI. ${ }^{4,5,7,8}$ The therapy options for ALI or pediatric ARDS (PARDS) in this vulnerable population are currently limited. ${ }^{5,9}$ Thus, investigations of effective therapeutic strategies to alleviate LPS-induced ALI in surfactant-insufficiency lungs are warranted.

Steroid has been long considered a classical therapy for neonatal ARDS due to its anti-inflammatory effect; however, the side effects of systemic steroids remain a major concern. ${ }^{10-12}$ For the prevention of side effects, several animal or clinical studies used intratracheal instillation ${ }^{13,14}$ or inhalation ${ }^{15-18}$ of steroids for the treatment of meconium aspiration syndrome (MAS) or prevention of bronchopulmonary dysplasia (BPD) and other respiratory morbidities. However, the local treatment with steroids remains challenging particularly in surfactant-deficiency lungs with alveolar collapse and diffuse atelectasis, which is a common problem in preterm infants ${ }^{7,19}$. To this end, intratracheal administration of surfactant is an effective treatment for surfactant-deficiency lung diseases or PARDS. ${ }^{19,20}$ Additionally, growing evidence suggests that surfactants can be used as a vehicle for intratracheal steroid administration. ${ }^{20}$ This delivery mode distributes the steroid well in the lungs without changing the biophysical and chemical properties of the drug. ${ }^{21-24}$ In animal studies, this steroid delivery mode has been used to investigate its efficacy in reducing ALI in animal models of surfactant-depleted lung diseases $,{ }^{23,25} \mathrm{MAS},{ }^{26}$ hyperoxia ${ }^{27}$, and injurious mechanical ventilation. ${ }^{28,29}$ In clinical studies, this steroid delivery mode has been used to prevent $\mathrm{BPD}^{30-32}$ and chronic diseases ${ }^{33}$ or to treat neonatal $\mathrm{ARDS}^{34,35}$ in premature infants. However, no study has been conducted to investigate the therapeutic effect of this steroid delivery approach on LPS-induced ALI in surfactant-insufficiency lungs.

This study aimed to investigate the therapeutic effects of intratracheally instilled budesonide (BUD) delivered by two concentrations of exogenous surfactant on LPS-induced ALI in surfactant-insufficiency rat lungs. Our ALI model was established by repeated saline lavage to produce surfactant insufficiency, followed by an intratracheal LPS insult.

\section{2 | MATERIALS AND METHODS}

\subsection{Ethic statement}

The experimental protocols described in this study were approved by the Institutional Animal Care and Use Committee of National Yang Ming Chiao Tung University, Taiwan (YMIACUC number: 981239) and were in accordance with the recommendation in the Guideline for the Care and Use of Laboratory Animals published by the Council of Agriculture Executive Yuan, Taiwan.

\section{2 | Animal preparation}

Male rats weighing 400-500 $\mathrm{g}$ at the age of 12 weeks received general anesthesia with isoflurane $2 \%$ at $2 \mathrm{~L} / \mathrm{min}$ $\mathrm{O}_{2}$ plus intraperitoneal injection with $25 \%$ urethane $(5 \mathrm{ml} / \mathrm{kg})$ before the surgical procedures. Anesthetic efficacy was determined by the lack of withdrawal from painful stimulus on the tail. All animals were placed in a supine position with a subcutaneous injection of lidocaine hydrochloride $(2 \%)$ for local anesthesia. A mid-cervical tracheostomy was performed using a 16-gauge cannula. A polyethylene tube was placed into the femoral artery for the continuous recording of arterial blood pressure and blood sampling. Another catheter was inserted into the femoral vein for intravenous infusion. After these procedures, all animals were paralyzed with an intravenous injection of cisatracurium besylate $(0.2 \mathrm{mg} / \mathrm{kg})$, followed by a continuous intravenous infusion of the same agent $(0.05 \mathrm{mg} / \mathrm{kg} / \mathrm{min})$. The animals were then sedated with a continuous intravenous infusion of propofol $(0.7 \mathrm{mg} / \mathrm{kg} / \mathrm{min})$. The animals were connected to a volume-controlled animal ventilator (Model 683, Harvard Apparatus, Holliston, MA, USA) to establish conventional ventilation. A tidal volume of $6 \mathrm{~mL} / \mathrm{kg}$, an inspiratory versus expiratory ratio of 1:1, a positive end-expiratory pressure (PEEP) of $3 \mathrm{~cm}$ $\mathrm{H}_{2} \mathrm{O}$, and a fractional $\mathrm{FiO}_{2}$ concentration of 1.0 were maintained throughout the experiments. The original ventilation rate was set at 60 breaths/min, followed by an increment or decrement of $2-3$ breaths/min 
to maintain an arterial blood $\mathrm{PCO}_{2}$ within 40 and 50 torr $(5.33-6.67 \mathrm{kPa})$ and $\mathrm{pH}>7.25$. The body temperature was maintained at $38{ }^{\circ} \mathrm{C}-39{ }^{\circ} \mathrm{C}$ via a servo-controlled heating blanket with a probe monitoring the anal temperature throughout the experiments.

\section{3 | Monitoring of physiological parameters}

During the experiment, electrocardiography, mean arterial blood pressure, and the percentage of arterial hemoglobin oxygen saturation $\left(\mathrm{SpO}_{2}\right)$ were continuously measured via a monitor (M1205A Omni-Care 24/24C, Hewlett Packard, Essex, MA, USA). Respiratory flow and airway pressure were measured with a heated pneumotachograph coupled to a differential pressure transducer (MP-45-16, Validyne, Kenilworth, NJ, USA) and a pressure transducer (MP45-28, Validyne), respectively. The tidal volume was integrated from the flow signal. These signals were recorded by a recorder (TA11, Gould, Eastlake, OH, USA) and a data acquisition system (PowerLab 16/30, ADInstruments Pty Ltd, Bella Vista, NSW, Australia). Arterial blood gas levels were analyzed using an autoanalyzer (OPTIMedical, Roswell, GA, USA). Alveolar-arterial oxygen gradient $\left(\mathrm{AaDO}_{2}\right)$ was calculated.

\section{4 | Surfactant depletion by lavage and LPS-induced lung injury}

After the baseline cardiopulmonary data had been collected, all rats received lung lavage with $10 \mathrm{~mL} / \mathrm{kg}$ normal saline intratracheally via a tracheostomy tube for both sides of the lungs. An additional saline lavage was given 5 min later if $\mathrm{Spo} 2$ remained above 90\%. When $\mathrm{PaO}_{2}$ dropped to less than 150 torr (20 kPa) (at $\mathrm{FiO}_{2}=1.0$ ), the rat was considered to have reached surfactant depletion. Subsequentially, a dose of $50 \mu \mathrm{g} / \mathrm{kg}$ LPS diluted by saline to a total volume of $1 \mathrm{~mL}$ was intratracheally instilled into the lungs to induce ALI. An additional 15 min period of postinjury stabilization was allowed before the subsequent procedures.

\section{5 | Animal groups}

The surfactant-depleted and LPS-injured rats were assigned randomly to one of the following five study groups ( $\mathrm{n}=5$ for each group) and received different intratracheal treatments with a total volume of 2 $\mathrm{mL} / \mathrm{kg}:$ 1) Control (no treatment); 2) intratracheal treatment with BUD alone (IT-NS-BUD; $0.5 \mathrm{mg} / \mathrm{kg}$ BUD in saline, AstraZeneca Taiwan, Taipei, Taiwan); 3) IT-DS-BUD (0.5 mg/kg BUD) in a solution of $10 \mathrm{mg} / \mathrm{mL}$ diluted surfactant; Survanta, Abbott Laboratories, Abbott Park, IL, USA); 4) IT-FS-BUD (0.5 mg/kg BUD in a solution of $25 \mathrm{mg} / \mathrm{mL}$ full-strength surfactant); 5) intratracheal treatment with full-strength surfactant alone (IT-FS; a solution of $25 \mathrm{mg} / \mathrm{mL}$ full-strength surfactant alone). These intratracheal treatments were performed by using a syringe pump to instill the solutions into a side port of the endotracheal tube adaptor for $10 \mathrm{~min}$.

\section{6 | Experimental protocol}

After starting the above-mentioned intratracheal treatments, the PEEP of the ventilator setting was turned up to $5 \mathrm{~cm} \mathrm{H}_{2} \mathrm{O}$. A total of $4 \mathrm{~h}$ was used for observation for each experiment. During the study period, sodium bicarbonate $(1-2 \mathrm{meq} / \mathrm{kg} / \mathrm{dose})$ was given to the animals displaying metabolic acidosis $(\mathrm{pH}<7.20)$ with a base excess lower than $-8 \mathrm{meq} / \mathrm{L}$. Epinephrine $(0.01 \mathrm{mg} / \mathrm{kg}$, one bolus injection) was given to the animals displaying bradycardia (heart rate $<100$ beats $/ \mathrm{min}$ ) every $3-5$ min if necessary. The arterial blood samples $(0.2 \mathrm{~mL})$ were obtained hourly for blood gas analysis (Chiron, Ciba Corning Diagnostics Corporation, MA, USA) at 0 and 30 min and then hourly until the end of experiment.

\section{7 | Pathological examinations}

At the end of the experiments, the animals were euthanized under deep anesthesia with a high dose of $15 \%$ potassium chloride $(3 \mathrm{~mL})$. Within $5 \mathrm{~min}$ after death, the ventilator was stopped, and a positive airway pressure equivalent to PEEP of $5 \mathrm{~cm} \mathrm{H}_{2} \mathrm{O}$ was applied. The chest wall was then opened, and the trachea was clamped. The airway and lung were incised and fixed in $10 \%$ formaldehyde solution for histological preparations and examinations. Routine techniques were used to prepare the lung tissues for paraffin embedding. Thin sections were stained with hematoxylin and eosin stain. The sections were examined under light microscopy (Olympus AX-80, Yuanyu Industry CO., Ltd, Taipei, Taiwan), and histology was scored using a 
quantitative system by an investigator blinded to the identity of the specimens. Injury scores were based on the following pathohistological characteristics: alveolar inflammation, interstitial inflammation, alveolar hemorrhage, interstitial hemorrhage, atelectasis, necrosis, and overdistension. Injury score was graded 0,1 , 2,3 , and 4 for abnormalities noted in $0 \%, 25 \%, 50 \%, 75 \%$, and diffusely covering the lung field, respectively. Multiple (>10) fields of the lung section for each pathohistological characteristic were examined, and the scores were averaged to obtain a mean value.

\section{8 | Immunohistochemical assessments}

Lung sections $(5 \mu \mathrm{m})$ from paraffin-embedded tissues were used for immunohistochemical studies. After deparaffinization and dehydration, endogenous peroxidases were blocked with $5 \% \mathrm{H}_{2} \mathrm{O}_{2}$, nonspecific proteinbinding sites were blocked with Thermo Scientific Ultra V block (TA-060-PBQ, ThermoFisher Scientific, Pittsburg, USA), and the slices were permeabilized with $0.5 \%$ Triton X-100 (Merck, Kenilworth, USA) in Tris-buffered saline. The slices were incubated with rabbit anti-myeloperoxidase or anti-tumor necrosis factor (TNF)- $\alpha$ primary antibody (CAT: Abcam, ab6671, 1:50; Abcam, ab9535, 1:100, Cambridge, MA, USA) overnight at $4{ }^{\circ} \mathrm{C}$. The subsequent steps of incubation included an enhancer reagent and horseradishperoxidase-linked secondary antibody, both of which are components of the Polink-2 Plus HRP Detection Kit (D39, GBI Labs, Bothell, WA, USA). The staining was visualized with diaminobenzidine. The slices were mounted on microscopic slides with Eukitß) (Merck, Kenilworth, NJ, USA) and digitally recorded with a microscope slide scanner (Zeiss Mirax Midi Slide Scanner, Carl Zeiss MicroImaging GmbH, Germany) operated by a CaseViewer software (3D Histech Ltd., Hungary).

Myeloperoxidase is a biomarker of neutrophil activation, and TNF- $\alpha$ is an important cytokine in LPSinduced lung inflammation. ${ }^{36,37}$ The levels of myeloperoxidase and TNF- $\alpha$ expression in lung sections were assessed with a semiquantitative approach. The percentage of positive staining cells per slide (0\% to $100 \%)$ was multiplied by the dominant intensity pattern of staining ( 0 , negative or trace; 1 , weak; 2 , moderate; 3 , intense). The maximal score was 300 . All the histological sections were examined by Aperio Color Deconvolution v9 (Leica Microsystems, Germany).

\section{9 | Statistical analysis}

Continuous data with a normal distribution are presented as mean \pm standard error of the mean (SEM). Ranked data or data that failed to follow a normal distribution are presented as medians with interquartile range. For within-group comparison, a paired Student t-test was used to compare cardiopulmonary data between preinjury and immediately post injury. For between-group comparison, one-way analysis of variance followed by post hoc Student-Newman-Keuls test was performed for the comparison continuous variables, and the Kruskal-Wallis test followed by pairwise comparison was conducted for the comparison of lung injury scores among the five study groups. Statistical significance was defined as p value less than 0.05. Two-tailed $\mathrm{p}$ values of less than 0.05 were considered statistically significant for all analyses. All data management and analyses were performed using Statistical Package for Social Science version 22 (SPSS Inc., Chicago, IL, USA).

\section{3 | RESULTS}

Table 1 shows the physiological conditions at pre- and post-injury (before intratracheal treatment). Severe acidosis with a concomitant increase in $\mathrm{PaCO}_{2}$ and a notable drop in $\mathrm{PaO}_{2}$ were found for all animals the after induction of ALI by lavage and LPS $(\mathrm{P}<0.05)$. All animal groups also presented significant increases in peak inspiratory pressure, $\mathrm{AaDO}_{2}$, and oxygen index after ALI. Except for the IT-DS-BUD group, all other groups showed a significant drop in the mean arterial blood pressure after ALI. However, these cardiopulmonary parameters at post-injury (before intratracheal treatment) showed no statistical difference among the five study groups.

After intratracheal treatments, the post-injury deteriorations of $\mathrm{PaO}_{2}$ and $\mathrm{AaDO}_{2}$ significantly improved over time in the IT-FS-BUD and IT-FS groups (Figures 1A and 1C). By contrast, these improvements were not observed in the Control, IT-NS-BUD, and IT-DS-BUD groups (Figures 1A and 1C). The base excess 
remained in the normal range during the study period in the IT-FS-BUD and IT-FS groups but worsened in the Control, IT-NS-BUD, and IT-DS-BUD groups (Figure 1D). The post-injury deteriorations of $\mathrm{PaCO}_{2}$ significantly improved over time in all the study groups (Figure 1B). The IT-FS-BUD and IT-FS groups also had a lower oxygen index (Figure 2A), higher mean arterial pressure (Figure 2B), and lower peak inspiratory pressure (Figure 2C) compared with the other three groups throughout the whole study period. Heart rate changed variedly over time among the five study groups, and no significance can be detected between any two groups at almost all time points (Figure 2D).

A histological evaluation of the lung sections from the Control and IT-NS-BUD groups revealed extensive inflammatory cell infiltration, hemorrhage, edema, and atelectasis, and all of these pathohistological changes were lessened in lung sections from the IT-DS-BUD, IT-FS-BUD, and IT-FS groups (Figure 3). These observations were confirmed by comparison of the group data in terms of lung injury scores for each pathohistological characteristic or total lung injury scores (Table 2). Numerically, the total injury scores in the five study groups followed the order IT-FS-BUD $<$ IT-DS-BUD or IT-FS $<$ IT-NS-BUD $<$ Control (Table 2). Further immunohistochemical staining of the lung sections from the Control and IT-FS groups revealed marked signals of myeloperoxidase and TNF- $\alpha$ in the alveoli space and interstitials of the alveoli (Figure 4). These signals were reduced in the lung sections from the IT-NS-BUD, IT-DS-BUD, and IT-FS-BUD groups (Figure 4). Comparisons of group data revealed that the immunostaining intensity of myeloperoxidase in the IT-NS-BUD, IT-DS-BUD, and IT-FS-BUD groups was significantly smaller than that in the Control and IT-FS groups (Figure 5). Additionally, the immunostaining intensity of TNF- $\alpha$ in the IT-FS-BUD group was significantly smaller than that in the other four groups (Figure 5).

\section{4 | DISCUSSION}

In this study, we investigated the therapeutic effect of BUD intratracheally delivered by two (diluted and full strength) concentrations of exogenous surfactant on the ALI in a rat model. The ALI in our model was established by repeated saline lavage, to produce surfactant insufficiency, followed by an intratracheal LPS insult. Our results demonstrated that intratracheal treatments with full-strength surfactant with or without BUD improved post-injury deteriorations of cardiopulmonary variables. Additionally, intratracheal treatments with surfactant (both concentrations) with or without BUD alleviated ALI, with IT-FS-BUD being the most effective treatment, as evidenced by our data of lung injury scores. Furthermore, intratracheal treatments with BUD delivered by normal saline or surfactant (both concentrations) ameliorated lung inflammation, with IT-FS-BUD being the most effective treatment, as evidenced by our data of immunohistochemical analysis. Collectively, among our therapeutic strategies, intratracheal BUD delivered by full-strength surfactant conferred the optimal protection against LPS-induced ALI in surfactant-insufficiency rat lungs.

Preterm infants may concurrently have surfactant deficiency and Gram-negative bacterial infections of the lungs, which are major causes of PARDS. ${ }^{4,5,7}$ To avoid the side effects of systemic steroid, ${ }^{10-12}$ several investigators advocated the use of intratracheal steroid for the treatment of ALI or PARDS. ${ }^{13-18}$ However, surfactant deficiency may lead to alveolar collapse and diffuse lung atelectasis, ${ }^{7,19}$ which hinders the uniform distribution of drug within the lungs and reduces the efficacy of local steroid treatment. Surfactant is a surface tension-lowering agent that has been widely used to treat surfactant-deficiency lung diseases or PARDS. ${ }^{8,19}$ When instilled into the trachea, the surfactant may spread throughout the lungs, driven by the surface tension gradient. ${ }^{20}$ For this reason, surfactant has been proposed as a vehicle for intratracheal steroid administration. ${ }^{20}$ This notion is supported by the stable biophysical and chemical properties of the surfactant/steroid mixtures under ex vivo condition ${ }^{21-23}$ and enhanced pulmonary distribution of steroid in vivo when using a surfactant as the vehicle. ${ }^{21-24}$ In our study, IT-FS provided certain benefits against ALI but was ineffective in reducing lung inflammation. On the other hand, IT-NS-BUD was ineffective in alleviating ALI but had a slight effect on the reduction of lung inflammation. Our findings regarding the superiority of IT-FS-BUD combined therapy in alleviating ALI and lung inflammation in our model provide a strong evidence for using full-strength surfactant as a vehicle for intratracheal steroid therapy.

The use of surfactant as a vehicle to deliver steroids to the lungs of premature infants has been the subject 
of increasing clinical interest. ${ }^{20}$ Several clinical studies have investigated the efficacy of this steroid delivery mode in preventing $\mathrm{BPD}^{32}$ and chronic respiratory diseases ${ }^{33}$ or in treating neonatal $\mathrm{ARDS}^{34,35}$ in premature infants. Two recent meta-analysis studies reported that intratracheal instillation of steroid-surfactant combination was an effective therapy for preventing BPD in preterm infants with neonatal ARDS, but its benefit in reducing mortality was inconsistent. ${ }^{30,31}$ Given the diverse etiology of PARDS, ${ }^{5,7}$ several animal studies have investigated the efficacy of using a surfactant as a vehicle for intratracheal steroids in reducing ALI in animal models of surfactant-depleted lung diseases, ${ }^{23,25} \mathrm{MAS},{ }^{26}$ hyperoxia ${ }^{27}$, and injurious mechanical ventilation..$^{28,29}$ Regardless of the type of insult to induce ALI, these studies reported that intratracheal instillation of steroid-surfactant combination can alleviate $\mathrm{ALI}^{23,25-29}$ and reduce lung inflammation. ${ }^{23,26-28}$ Our model is a type of ALI in surfactant-insufficiency lungs, particularly with lung inflammation induced by LPS. Our results demonstrated for the first time that intratracheal steroid-surfactant therapy is promising in this particular setting.

This study exhibited several limitations. First, we did not use preterm rats as our animal model for lung surfactant deficiency because preterm rats have a high mortality rate in response to the insult of LPS when they are mechanically ventilated. Second, our observation period was $4 \mathrm{~h}$, and thus, we cannot be certain about the changes that might have occurred after that time. Third, the concentration of BUD in the systemic circulation was not measured, and thus, further studies targeting this topic are required.

In conclusion, intratracheal BUD delivered by full-strength surfactant effectively alleviates ALI and lung inflammation in surfactant-insufficiency rat lungs with LPS insult. This intratracheal steroid-surfactant treatment may be considered a potential therapy for preterm infants with Gram-negative bacteria lung infections.

\section{ACKNOWLEDGMENTS}

The authors are grateful to the KGSupport Academic Submission Services for assisting with language editing. This study was supported by grants from the Ministry of Science and Technology, Taiwan (MOST 105-2314B-010-044 and MOST 107-2320-B-010-027-MY3) and Taipei Veterans General Hospital, Taiwan (V110C-070 and V110C-170).

\section{CONFLICT OF INTEREST}

All authors declare that they have no conflict of interest.

\section{REFERENCES}

1. Black RE, Morris SS, Bryce J. Where and why are 10 million children dying every year? Lancet . 2003;361:2226-2234.

2. Duke T. Neonatal pneumonia in developing countries. Arch Dis Child Fetal Neonatal Ed . 2005;90:F211-F219.

3. Mortality GBD and Causes of Death C. Global, regional, and national life expectancy, all-cause mortality, and cause-specific mortality for 249 causes of death, 1980-2015: a systematic analysis for the Global Burden of Disease Study 2015. Lancet . 2016;388:1459-1544.

4. Chakraborty M, McGreal EP, Kotecha S. Acute lung injury in preterm newborn infants: mechanisms and management. Paediatr Respir Rev . 2010;11:162-170.

5. Heidemann SM, Nair A, Bulut Y, Sapru A. Pathophysiology and Management of Acute Respiratory Distress Syndrome in Children. Pediatr Clin North Am . 2017;64:1017-1037.

6. Sugitharini V, Pavani K, Prema A, Berla Thangam E. TLR-mediated inflammatory response to neonatal pathogens and co-infection in neonatal immune cells. Cytokine . 2014;69:211-217.

7. Martin R. Pathophysiology, clinical manifestations, and diagnosis of respiratory distress syndrome in the newborn. In: UpToDate, Garcia-Prats, JA (Ed), UpToDate, Waltham, MA, USA, 2020.

8. Sardesai S, Biniwale M, Wertheimer F, Garingo A, Ramanathan R. Evolution of surfactant therapy for respiratory distress syndrome: past, present, and future. Pediatr Res . 2017;81:240-248.

9. Pediatric Acute Lung Injury Consensus Conference G. Pediatric acute respiratory distress syndrome: 
consensus recommendations from the Pediatric Acute Lung Injury Consensus Conference. Pediatr Crit Care Med . 2015;16:428-439.

10. Yehya N, Servaes S, Thomas NJ, Nadkarni VM, Srinivasan V. Corticosteroid exposure in pediatric acute respiratory distress syndrome. Intensive Care Med . 2015;41:1658-1666.

11. Doyle LW, Cheong JL, Ehrenkranz RA, Halliday HL. Early ( $<8$ days) systemic postnatal corticosteroids for prevention of bronchopulmonary dysplasia in preterm infants. Cochrane Database Syst Rev . 2017;10:CD001146.

12. Monteverde-Fernandez N, Cristiani F, McArthur J, Gonzalez-Dambrauskas S. Steroids in pediatric acute respiratory distress syndrome. Ann Transl Med . 2019;7:508.

13. Mokra D, Mokry J, Drgova A, Petraskova M, Bulikova J, Calkovska A. Intratracheally administered corticosteroids improve lung function in meconium-instilled rabbits. J Physiol Pharmacol . 2007;58 Suppl 5:389-398.

14. Lin CH, Jeng MJ, Yang YC, Hsiao YH, Kou YR. Comparison of different dosing strategies of intratracheally instilled budesonide on meconium injured piglet lungs. Pediatr Pulmonol . 2017;52:891-899.

15. Bassler D, Plavka R, Shinwell ES, Hallman M, Jarreau PH, Carnielli V, Van den Anker JN, Meisner C, Engel C, Schwab M, Halliday HL, Poets CF, Group NT. Early inhaled budesonide for the prevention of bronchopulmonary dysplasia. N Engl J Med . 2015;373:1497-1506.

16. Tukova J, Smisek J, Zlatohlavkova B, Plavka R, Markova D. Early inhaled budesonide in extremely preterm infants decreases long-term respiratory morbidity. Pediatr Pulmonol . 2020;55:1124-1130.

17. Bassler D, Shinwell ES, Hallman M, Jarreau PH, Plavka R, Carnielli V, Meisner C, Engel C, Koch A, Kreutzer K, van den Anker JN, Schwab M, Halliday HL, Poets CF; Neonatal European Study of Inhaled Steroids Trial Group. Long-term effects of inhaled budesonide for bronchopulmonary dysplasia. N Engl J Med . 2018;378:148-157.

18. Shah SS, Ohlsson A, Halliday HL, Shah VS. Inhaled versus systemic corticosteroids for preventing bronchopulmonary dysplasia in ventilated very low birth weight preterm neonates. Cochrane Database Syst Rev . 2017;10:CD002058.

19. Chakraborty M, Kotecha S. Pulmonary surfactant in newborn infants and children. Breathe . 2013;9:476-488.

20. Boel L, Banerjee S, Chakraborty M. Postnatal steroids in extreme preterm infants: Intra-tracheal instillation using surfactant as a vehicle. Paediatr Respir Rev . 2018;25:78-84.

21. Chen CM, Chang $\mathrm{CH}$, Chao $\mathrm{CH}$, Wang MH, Yeh TF. Biophysical and chemical stability of surfactant/budesonide and the pulmonary distribution following intra-tracheal administration. Drug Deliv . 2019;26:604-611.

22. Nimmo AJ, Carstairs JR, Patole SK, Whitehall J, Davidson K, Vink R. Intratracheal administration of glucocorticoids using surfactant as a vehicle. Clin Exp Pharmacol Physiol . 2002;29:661-665.

23. Ricci F, Catozzi C, Ravanetti F, Murgia X, D'Alo F, Macchidani N, Sgarbi E, Di Lallo V, Saccani F, Pertile M, Cacchioli A, Catinella S, Villetti G, Civelli M, Amadei F, Stellari FF, Pioselli B, Salomone F. In vitro and in vivo characterization of poractant alfa supplemented with budesonide for safe and effective intratracheal administration. Pediatr Res . 2017;82:1056-1063.

24. Huang LT, Yeh TF, Kuo YL, Chen PC, Chen CM. Effect of surfactant and budesonide on the pulmonary distribution of fluorescent dye in mice.Pediatr Neonatol . 2015;56:19-24.

25. Yang CF, Jeng MJ, Soong WJ, Lee YS, Tsao PC, Tang RB. Acute pathophysiological effects of intratracheal instillation of budesonide and exogenous surfactant in a neonatal surfactant-depleted piglet model. Pediatr Neonatol . 2010;51:219-226.

26. Lin CH, Jeng MJ, Kuo BI, Kou YR. Effects of surfactant lavage combined with intratracheal budesonide instillation on meconium-injured piglet lungs. Pediatr Crit Care Med . 2016;17:e287-95.

27. Gie AG, Regin Y, Salaets T, Casiraghi C, Salomone F, Deprest J, Vanoirbeek J, Toelen J. Intratracheal budesonide/surfactant attenuates hyperoxia-induced lung injury in preterm rabbits. Am J Physiol Lung Cell Mol Physiol . 2020;319:L949-L956.

28. Hillman NH, Kothe TB, Schmidt AF, Kemp MW, Royse E, Fee E, Salomone F, Clarke MW, Musk GC, Jobe AH. Surfactant plus budesonide decreases lung and systemic responses to injurious ventilation in 
preterm sheep.Am J Physiol Lung Cell Mol Physiol . 2020;318:L41-L48.

29. Ikegami M, Berry D, elKady T, Pettenazzo A, Seidner S, Jobe A. Corticosteroids and surfactant change lung function and protein leaks in the lungs of ventilated premature rabbits. J Clin Invest . 1987;79:1371-1378.

30. Venkataraman R, Kamaluddeen M, Hasan SU, Robertson HL, Lodha A. Intratracheal administration of budesonide-surfactant in prevention of bronchopulmonary dysplasia in very low birth weight infants: a systematic review and meta-analysis. Pediatr Pulmonol . 2017;52:968-975.

31. Zhong YY, Li JC, Liu YL, Zhao XB, Male M, Song DK, Bai Y. Early intratracheal administration of corticosteroid and pulmonary surfactant for preventing bronchopulmonary dysplasia in preterm infants with neonatal respiratory distress syndrome: a meta-analysis. Curr Med Sci . 2019;39:493-499.

32. McEvoy CT, Ballard PL, Ward RM, Rower JE, Wadhawan R, Hudak ML, Weitkamp JH, Harris J, Asselin J, Chapin C, Ballard RA. Dose-escalation trial of budesonide in surfactant for prevention of bronchopulmonary dysplasia in extremely low gestational age high-risk newborns (SASSIE). Pediatr Res . 2020;88:629-636.

33. Yeh TF, Lin HC, Chang CH, Wu TS, Su BH, Li TC, Pyati S, Tsai CH. Early intratracheal instillation of budesonide using surfactant as a vehicle to prevent chronic lung disease in preterm infants: a pilot study.Pediatrics . 2008;121:e1310-1318.

34. Deliloglu B, Tuzun F, Cengiz MM, Ozkan H, Duman N. Endotracheal surfactant combined with budesonide for neonatal ARDS. Front Pediatr . 2020;8:210.

35. Kothe TB, Sadiq FH, Burleyson N, Williams HL, Anderson C, Hillman NH. Surfactant and budesonide for respiratory distress syndrome: an observational study. Pediatr Res . 2020;87:940-945.

36. Haegens A, Heeringa P, van Suylen RJ, Steele C, Aratani Y, O’Donoghue RJ, Mutsaers SE, Mossman BT, Wouters EF, Vernooy JH. Myeloperoxidase deficiency attenuates lipopolysaccharide-induced acute lung inflammation and subsequent cytokine and chemokine production. J Immunol. 2009;182:79907996.

37. Knapp S, Florquin S, Golenbock DT, van der Poll T. Pulmonary lipopolysaccharide (LPS)-binding protein inhibits the LPS-induced lung inflammation in vivo. J Immunol . 2006;176:3189-3195.

\section{FIGURE LEGENDS}

FIGURE 1 . Changes in $\mathrm{PaO}_{2}$ (A), $\mathrm{PaCO}_{2}$ (B), $\mathrm{AaDO}_{2}$ (C), and base excess (D) over the 4 h post-injury period after different intratracheal treatments in the five study groups. Control group, no treatment; ITNS-BUD group, treatment with BUD $(0.5 \mathrm{mg} / \mathrm{kg})$ in saline; IT-DS-BUD group, treatment with BUD $(0.5$ $\mathrm{mg} / \mathrm{kg})$ in a solution of diluted Survanta $(10 \mathrm{mg} / \mathrm{mL})$; IT-FS-BUD group, treatment with BUD $(0.5 \mathrm{mg} / \mathrm{kg})$ in a solution of full-strength Survanta $(25 \mathrm{mg} / \mathrm{mL})$; IT-FS group, treatment with a solution of full-strength Survanta $(25 \mathrm{mg} / \mathrm{mL})$ alone. $\mathrm{PaO}_{2}$, oxygen tension in arterial blood; $\mathrm{PaO}_{2}$, carbon dioxide tension in arterial blood; $\mathrm{AaDO}_{2}$, alveolar-arterial oxygen gradient.

${ }^{\mathrm{a}} \mathrm{p}<0.05$ versus control group; ${ }^{\mathrm{b}} \mathrm{p}<0.05$ versus IT-NS-BUD group; ${ }^{\mathrm{c}} \mathrm{p}<0.05$ versus IT-FS-BUD group; ${ }^{\mathrm{d}} \mathrm{p}$ $<0.05$ versus IT-DS-BUD group. Data in each group $(\mathrm{n}=5)$ are expressed as mean \pm SEM.

FIGURE 2 . Changes in oxygen index (A), mean arterial pressure (B), peak inspiratory pressure (C), and heart rate (D) over the $4 \mathrm{~h}$ post-injury period after different intratracheal treatments in the five study groups. Control group, no treatment; IT-NS-BUD group, treatment with BUD $(0.5 \mathrm{mg} / \mathrm{kg})$ in saline; IT-DSBUD group, treatment with BUD $(0.5 \mathrm{mg} / \mathrm{kg})$ in a solution of diluted Survanta $(10 \mathrm{mg} / \mathrm{mL})$; IT-FS-BUD group, treatment with BUD $(0.5 \mathrm{mg} / \mathrm{kg})$ in a solution of full-strength Survanta $(25 \mathrm{mg} / \mathrm{mL})$; IT-FS group, treatment with a solution of full-strength Survanta $(25 \mathrm{mg} / \mathrm{mL})$ alone.

${ }^{\mathrm{a}} \mathrm{p}<0.05$ versus control group; ${ }^{\mathrm{b}} \mathrm{p}<0.05$ versus IT-NS-BUD group; ${ }^{\mathrm{p}} \mathrm{p}<0.05$ versus IT-FS-BUD group; ${ }^{\mathrm{d}} \mathrm{p}$ $<0.05$ versus IT-DS-BUD group. Data in each group $(\mathrm{n}=5)$ are expressed as mean \pm SEM.

FIGURE 3 . Representative pulmonary histological photomicrographs of the five study groups with different intratracheal treatments. Lung sections were stained with hematoxylin and eosin. Control group, no treatment; IT-NS-BUD group, treatment with BUD $(0.5 \mathrm{mg} / \mathrm{kg})$ in saline; IT-DS-BUD group, treatment 
with BUD $(0.5 \mathrm{mg} / \mathrm{kg})$ in a solution of diluted Survanta $(10 \mathrm{mg} / \mathrm{mL})$; IT-FS-BUD group, treatment with BUD $(0.5 \mathrm{mg} / \mathrm{kg})$ in a solution of full-strength Survanta $(25 \mathrm{mg} / \mathrm{mL})$; IT-FS group, treatment with a solution of full-strength Survanta $(25 \mathrm{mg} / \mathrm{mL})$ alone.

FIGURE 4 . Representative pulmonary photomicrographs of immunohistochemical expression of myeloperoxidase (A) and TNF- $\alpha$ (B) in the five study groups with different intratracheal treatments. Myeloperoxidase is a marker of neutrophil activation, whereas TNF- $\alpha$ is an inflammatory cytokine. Control group, no treatment; IT-NS-BUD group, treatment with BUD $(0.5 \mathrm{mg} / \mathrm{kg})$ in saline; IT-DS-BUD group, treatment with BUD $(0.5 \mathrm{mg} / \mathrm{kg})$ in a solution of diluted Survanta $(10 \mathrm{mg} / \mathrm{mL})$; IT-FS-BUD group, treatment with BUD $(0.5 \mathrm{mg} / \mathrm{kg})$ in a solution of full-strength Survanta $(25 \mathrm{mg} / \mathrm{mL})$; IT-FS group, treatment with a solution of full-strength Survanta $(25 \mathrm{mg} / \mathrm{mL})$ alone.

FIGURE 5. Semiquantitative analysis of immunohistochemical expression of myeloperoxidase and TNF- $\alpha$ in lung sections from the five study groups. The percentage of positive staining cells per slide $(0 \%$ to $100 \%)$ was multiplied by the dominant intensity pattern of staining ( 0 , negative or trace; 1 , weak; 2 , moderate; 3 , intense). The maximal score was 300 .

${ }^{*} \mathrm{p}<0.05$ versus control group.

\section{Hosted file}

Table 1.pdf available at https://authorea.com/users/370894/articles/512951-efficacy-ofintratracheal-budesonide-surfactant-combined-therapy-in-surfactant-insufficient-ratlungs-with-lipopolysaccharide-insult

\section{Hosted file}

Table 2.pdf available at https://authorea.com/users/370894/articles/512951-efficacy-ofintratracheal-budesonide-surfactant-combined-therapy-in-surfactant-insufficient-ratlungs-with-lipopolysaccharide-insult

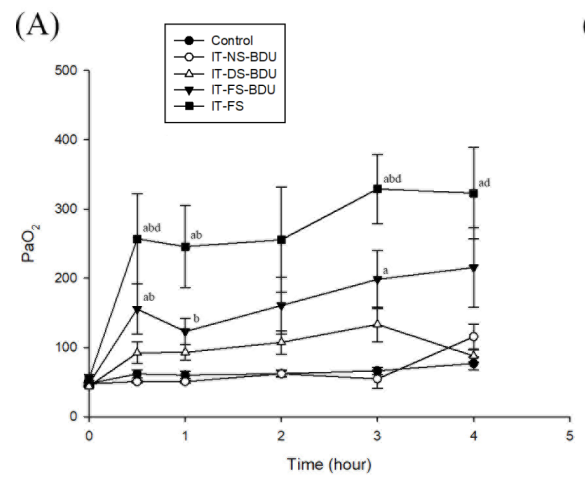

(C)

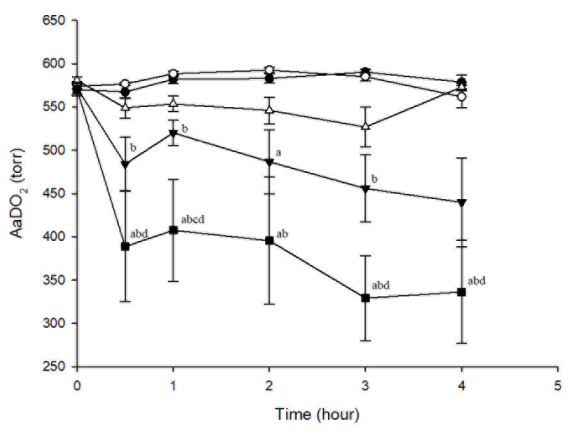

(B)

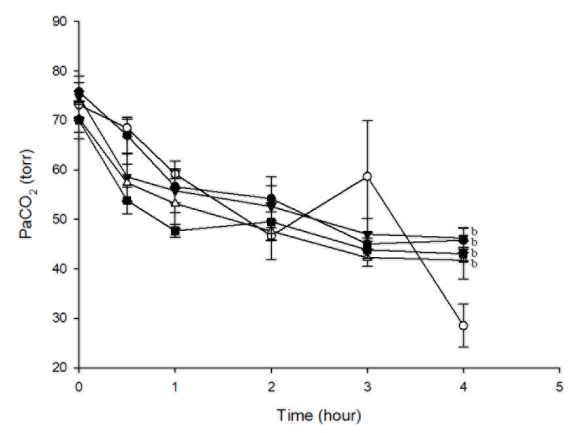

(D)

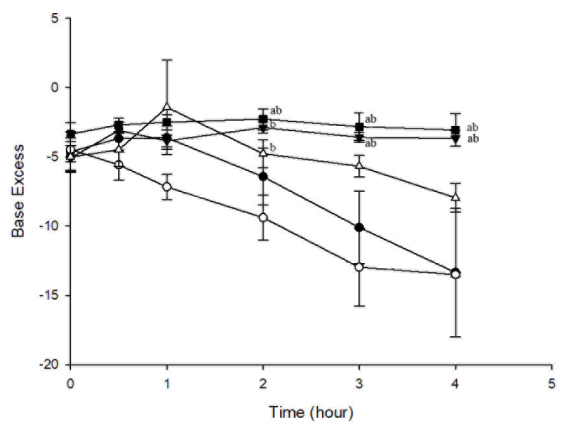



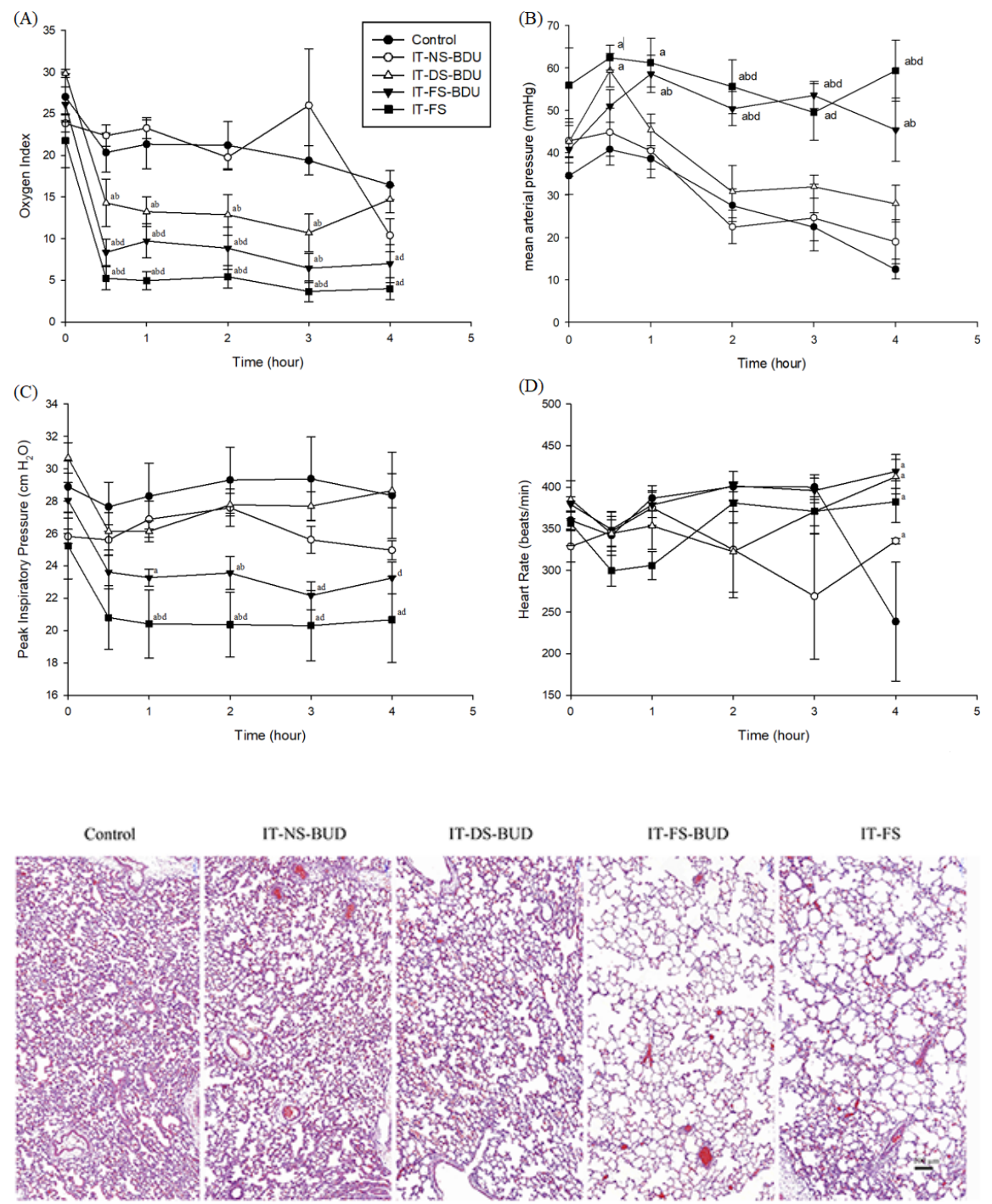

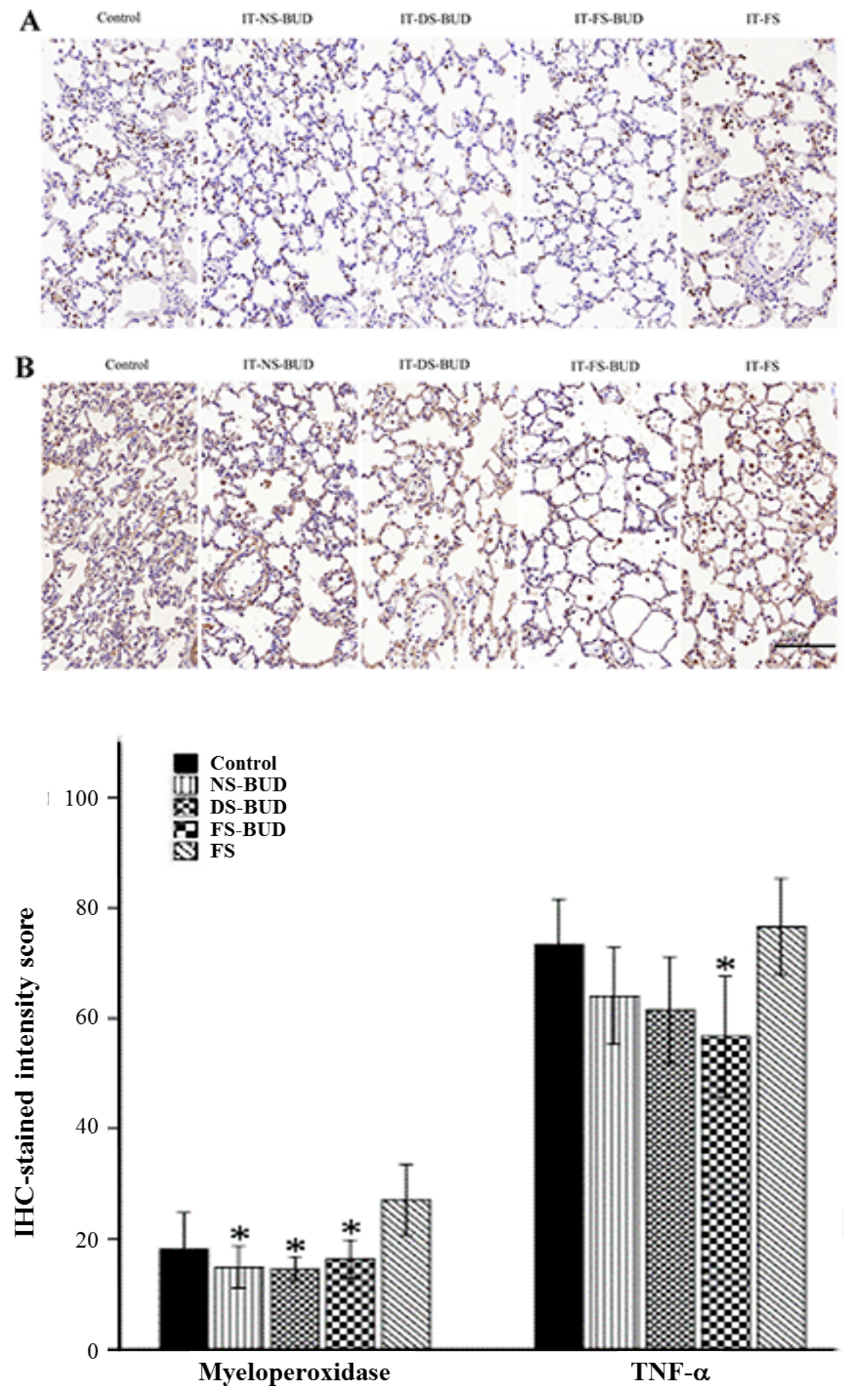